\title{
EVALUACIÓN PRELIMINAR DE LA MORTANDAD DE MASTOFAUNA NATIVA POR COLISIÓN CON VEHÍCULOS EN TRES RUTAS ARGENTINAS
}

\section{PRELIMINARY EVALUATION OF NATIVE MASTOFAUNA MORTALITY DUE TO COLLISION WITH VEHICLES IN THREE ARGENTINIAN ROUTES}

\author{
Fernando R. Barri ${ }^{1}$
}

\begin{abstract}
Resumen
La mortandad de fauna silvestre por colisión con vehículos en rutas ha sido ampliamente estudiada en países desarrollados. Esta información llevó a tomar medidas de prevención y mitigación. Sin embargo, en regiones como Latinoamérica es aún poco lo que se conoce al respecto. Se realizó un relevamiento preliminar para determinar el número de especies e individuos de la mastofauna silvestre mediana y grande hallados muertos por colisión con vehículos, en tres tramos de rutas nacionales argentinas colindantes con áreas naturales, durante 24 hs. Basándose en lo observado en este estudio sobre la problemática abordada, y la revisión bibliográfica, se sugieren además algunas futuras líneas de investigación a profundizar.
\end{abstract}

Palabras clave: Argentina, colisión con vehículos en rutas, mastofauna mediana y grande.

\begin{abstract}
Road kills of wild fauna has been extensively studied in developed countries. This information led to take prevention and mitigation measures. However, in regions such as Latin America the knowledge on this issue is scarce. We have conducted a preliminary survey to determine the number of species of wild mammal fauna of medium and large size found dead by collision with vehicles, in three stretches of Argentine national routes adjacent to natural areas for 24 hours. Based on what we observed, and the literature review, we also suggest some future research lines to be followed.
\end{abstract}

Key words: Argentina, road kills, medium and large mammal fauna.

\section{Introducción.}

La colisión con vehículos en rutas y caminos constituyen un serio problema para la conservación de los vertebrados terrestres (Spellerberg, 1998; Forman et al., 2003), siendo una de las causas de mortalidad más graves para varias especies animales alrededor del mundo (Lodé, 2000; Kerley et al., 2002). Las rutas actúan como barreras artificiales para la vida silvestre, impidiendo o limitando la dispersión de individuos entre poblaciones, lo que provoca aislamientos y reducción del flujo génico, y por lo tanto aumenta el riesgo de extinción local (Strasburg, 2006). Estas barreras potencian a su vez el efecto de la fragmentación del hábitat, una de las principales causas de pérdida de biodiversidad a nivel mundial (Sala et al., 2000), que para el caso de algunos mamíferos provoca entre el 65 al 85\% de las amenazas de extinción (Wilcove et al., 1998).

El estudio del impacto de las rutas sobre la vida silvestre tiene una larga tradición en el hemisferio norte (v. g. Caro et al. 2000; Joyce \& Mahoney, 2001; Baker et al., 2004; Taylor \& Goldingay, 2004; Blanco et al., 2005; Grilo et al., 2009). Asimismo, el incremento de la construcción de caminos en áreas naturales ha aumentado considerablemente la tasa de colisiones de vehículos con la vida silvestre (Forman et al., 2003). Los mamíferos grandes y medianos son particularmente vulnerables a este tipo de mortalidad, debido a su baja densidad y fecundidad, y sus amplias áreas de acción y movimientos (Spellerberg, 1998; Gittleman et al., 2001). En algunos grupos como los carnívoros, la colisión con vehículos puede ser la principal causa de mortalidad local (Clarke et al., 1998; Ferreras et al., 2001; Luell et al., 2003).

Por su parte, en Latinoamérica existen algunas estimaciones parciales sobre el efecto de las rutas en la mortandad de fauna silvestre y la supervivencia de sus poblaciones (v. g. Ledec \& Posas, 2003; Fischer et al., 2004; Araujo Bagno et al., 2004). En Argentina sólo se han realizado algunas aproximaciones a esta problemática (v. g. Trejo \& Seijas, 2003; Cairo \& Zalba, 2007; Martino et al., 2008), considerándose un factor importante a tener en cuenta para la conservación de especies vulnerables como los ciervos autóctonos (Dellafiore \& Maceira, 1998; Pereda \& Moreno, 2000), aunque aún es mucha la falta de información que existe en este sentido, más aún teniendo en cuenta que la misma es de vital 
importancia para el desarrollo de futuras medidas de prevención y mitigación.

En este estudio se realizó una evaluación preliminar del número de individuos de la mastofauna nativa muertos en un día por colisión contra vehículos, en tres tramos de rutas nacionales colindantes con áreas silvestres de Argentina. A la vez, se realizan consideraciones sobre futuros estudios que se deberían encarar para ampliar el conocimiento sobre la temática abordada.

\section{Materiales y métodos.}

Lugares de estudio.

Se seleccionaron tres tramos de similar longitud y tráfico vehicular (aproximadamente 3000 vehículos/día, Dirección Nacional de Vialidad Argentina, 2010) en distintas rutas nacionales argentina ubicadas en las regiones sur, centro y norte del país, colindantes en parte de su trayectoria con áreas naturales protegidas. Las rutas y tramos que se recorrieron fueron: (1) Ruta Nacional $\mathrm{N}^{\circ} 152$, entre General Acha y el Parque Nacional Lihuel Calel, en la provincia de la Pampa, de $120 \mathrm{~km}$ de extensión, en octubre de 2008; (2) Ruta Nacional $\mathrm{N}^{\circ} 510$, entre Valle Fértil y el Parque Provincial Ischigualasto, en la provincia de San Juan, de 100 km de extensión, en abril de 2009; y (3) Ruta Nacional $N^{\circ} 9$ Norte, entre Jesús María y la Reserva Natural Cerro Colorado, en la provincia de Córdoba, de 110 km de extensión, en agosto de 2009.

Métodos.

Cada tramo de las rutas seleccionadas se recorrió en vehículo a una velocidad de entre 10-20 kilómetros por hora por debajo de la mínima permitida $(60 \mathrm{~km} / \mathrm{h}$ en rutas nacionales), en un lapso de 24 hs entre ida y vuelta. Para ello, previo examinar el pronóstico meteorológico de manera de asegurarse recorrer los tramos de las rutas seleccionadas en días soleados con buena visibilidad, se partió a las 9 hs de la mañana del primer día y se registraron en planillas todos los individuos muertos a lo largo del trayecto de ida, estableciendo su posición específica dentro del kilometraje recorrido, y marcando además su ubicación con GPS. Posteriormente, a las 9 hs de la mañana del día siguiente se procedió a recorrer el mismo tramo en el sentido contrario o de regreso, registrando nuevamente los individuos muertos por colisión con vehículos a lo largo del tramo de la ruta nacional, utilizando la misma metodología. Así, para evaluar la mortandad de componentes 86 de la fauna de mamíferos nativos en los tres tramos de cada una de las rutas nacionales relevadas, sólo se tomaron en cuenta aquellos individuos encontrados en el tramo de regreso, de manera de asegurarse que en el análisis sólo se tuviera en cuenta el número de especies e individuos que fueron muertos por colisión con vehículos en ellas a lo largo de un día.
En cada caso el relevamiento se realizó con dos observadores, que fueron registrando a cada lado del vehículo los mamíferos nativos medianos y grandes muertos por colisión a lo largo de cada tramo de ruta. Una vez detectado un individuo muerto por colisión, se detuvo el vehículo y se procedió a su identificación, teniendo como base ante dudas taxonómicas el último libro publicado por la Sociedad Argentina para el Estudio de los Mamíferos al respecto (Barquez et al., 2006), tratando de determinar en cada caso el sexo y la edad aproximada (establecida como cría, juvenil, adulto). Por su parte, debido a la mayor dificultad de visualizarlos, y el consiguiente riesgo de subestimar la muestra, así como de establecer efectivamente su taxonomía, se descartaron del registro de esta evaluación aquellos mamíferos pequeños, fundamentalmente roedores, que se pudieran haber detectado a lo largo del recorrido en cada uno de los tramos de las tres rutas seleccionadas. También se descartaron de este primer registro aquellas otras especies de mamíferos no nativos (v. g. Lepus europaeus) y otros vertebrados hallados muertos como aves, reptiles o anfibios.

\section{Resultados y discusión.}

El resultado del relevamiento preliminar realizado sobre la mortandad de mamíferos nativos por colisión con vehículos en cada una de las tres rutas estudiadas puede observarse en la Tabla 1, en la que se incluye además el status de conservación para Argentina de cada especie de mamífero mediano y grande registrado (Barquez et al., 2006). Si bien en este relevamiento preliminar no se pudo evaluar las fluctuaciones intra e interanuales y su influencia sobre las actividad de los individuos, debido a la magnitud que implican este tipo de estudios (que requieren un gran esfuerzo de muestreo para estimar el número medio de individuos muertos por especie y por año para cada tramo de ruta), se puede estimar que el efecto de las rutas de Argentina colindantes a áreas silvestres sobre la mastofauna nativa es potencialmente negativo. Ello debido a que la colisión por vehículos se convierte en un factor extra de mortalidad dentro de la dinámica poblacional de las especies silvestres, que en algunos casos puede incrementar el riesgo de extinción local (Spellerberg, 1998; Lodé, 2000).

Este relevamiento preliminar sugiere además que la colisión con vehículos podría representar un peligro para la conservación de algunas poblaciones silvestres de mamíferos medianos y grandes de Argentina que se encuentran en peligro (v. g. gato montés, zorro colorado, zorro chilla, mara). En especies de mamíferos cuyas poblaciones se encuentran muy reducidas existe abundante evidencia que señala que para mantener su viabilidad poblacional es tan o más importante que la restauración del hábitat, reducir los factores de riesgo de mortalidad producidos causas 
Tabla 1. Número de individuos, por especie, registrados muertos tras colisionar contra vehículos en tres tramos de rutas nacionales de Argentina colindantes con áreas naturales protegidas, en un lapso de 24 hs.

\begin{tabular}{|c|c|c|c|}
\hline Especie y status de conservación & $\begin{array}{c}\text { RN } 152 \\
\text { (G. Acha - PN Lihuel Calel) }\end{array}$ & $\begin{array}{c}\text { RN } 510 \\
\text { (V. Fértil - PP Ischigualasto) }\end{array}$ & $\begin{array}{c}\text { RN } 9 \\
\text { (J. María - RN Cerro Colorado) }\end{array}$ \\
\hline Chaetophractus villosus (PM) & & 1 (hembra adulta) & 1 (macho adulto) \\
\hline Zaedyus pichiy (PM) & 1 (hembra adulta) & & \\
\hline Didelphis albiventris (PM) & & & 1 (hembra adulta) \\
\hline Conepatus chinga (PV) & 1 (macho adulto) & 1 (macho juvenil) & \\
\hline Dolichotis patagonum (VU) & & 1 (hembra juvenil) & \\
\hline Pseudalopex griseus (EP) & & 1 (macho adulto) & \\
\hline Pseudalopex gymnocercus (PM) & & & 1 (hembra adulta) \\
\hline Pseudalopex culpaeus (VU) & 1 (macho juvenil) & & \\
\hline Oncifelis geoffroyi (PV) & & & 1 (macho juvenil) \\
\hline Lama guanicoe (PV) & 1 (macho juvenil) & & \\
\hline
\end{tabular}

PM: precaución menor, PV: potencialmente vulnerable, VU: vulnerable, EP: en peligro

antrópicas, como es el caso de la colisión en rutas (v. g. Ferreras et al., 2001; Kerley et al., 2002). Por su parte, en el caso de aquellas especies de mamíferos con mayores densidades y distribución regional (v. g. zorrino, comadreja común, guanaco), como se ha sugerido (Strasburg, 2006) el efecto barrera que provocan las rutas sumado a la fragmentación del hábitat podría incrementar el aislamiento entre sus poblaciones, particularmente en algunas regiones como el norte de la provincia de Córdoba, que ha sufrido una de las tasas de deforestación más altas del mundo durante la última década (Zak et al., 2008).

Si bien es conocido que el número de animales que mueren en las rutas depende de la densidad de sus poblaciones, su biología, tipo de hábitat y estructura del paisaje, así como las características de la ruta y el tráfico que circula por ellas (Spellerberg, 1998; Forman et al., 2003; Malo et al., 2004), es preciso tomar nota de este tipo de evaluaciones preliminares, de manera de planificar estrategias que tiendan a reducir el efecto negativo de las rutas para la conservación de las poblaciones silvestres de nuestra mastofauna nativa.

En países desarrollados el trabajo mancomunado de biólogos e ingenieros ha intentado responder en las últimas décadas a esta problemática, buscando la manera de facilitar el movimiento de animales a través de corredores, pasos de fauna y el desarrollo de una serie de posibles soluciones prácticas para mitigar el efecto de las rutas sobre la fauna silvestre (v. g. Luell et al., 2003; Dodd et al., 2004; Seiler, 2005; Brodziewska, 2006; Iglesias Merchán, 2007). De allí la importancia de profundizar las investigaciones tendientes a determinar cuáles son los factores que incrementan el riesgo de colisiones y las medidas que podrían llevarse a cabo para mitigar el efecto negativo de las rutas sobre la vida silvestre de Argentina.
Teniendo en cuenta la escasa información con que se cuenta en Argentina en relación al impacto sobre la fauna silvestre de la colisión con vehículos, en base de lo observado en este estudio preliminar, se sugiere que entre los futuros estudios a profundizar deberían considerarse:

- Cuantificar el número de individuos muertos por especie y por año (incluyendo las variaciones interanuales), en las distintas rutas nacionales del país, especialmente en aquellas rutas colindantes a áreas protegidas o reservas.

- Determinar cuáles especies son más vulnerables a colisionar con vehículos, y cuáles las características biológicas que influyen en ello (comportamiento, tamaño, dieta, movimientos, áreas de acción, etc.).

- Precisar la magnitud del efecto barrera (cómo afecta la movilidad de los individuos, así como la distribución y dinámica de las poblaciones) que pueden provocar las rutas colindantes a áreas silvestres. En especial para aquellas especies en peligro que hayan sufrido un alto grado de fragmentación del hábitat, como el caso de Pseudalopex griseus, Oncifelis geoffroyi o Dolichotis patagonum.

- Establecer los factores que influyen en el número de individuos y especies muertas en las rutas, incluyendo las características estructurales de la ruta (diseño, señalización, barreras y/o pasos de fauna si los hubiera, etc.), la velocidad del vehículo, el volumen del tráfico, la topografía local, la accesibilidad a la ruta por parte de los animales desde sectores colindantes, etc. 
- Identificar sectores y características particulares de rutas colindantes a las áreas protegidas de Argentina que incrementan el riego de colisiones con la fauna silvestre.

Una vez que se cuente con esta información, así como con datos poblacionales cuantitativos de base para establecer la vulnerabilidad de las poblaciones o el riesgo de extinción, se podrán realizar proyecciones y modelos predictivos que establezcan los riesgos para la conservación de la fauna nativa a medida que se incremente la construcción de nuevas rutas y el tráfico vehicular, así también como sugerir las medidas de manejo necesarias para reducir o mitigar su impacto.

\section{Agradecimientos.}

J. Spicogna colaboró en el relevamiento de datos. D. Tamburini aportó valiosos comentarios al manuscrito.

\section{Literatura citada.}

Araujo Bagno M., Guimaraes Rodrigues F.H., Prada Villalobos M., Dalponte J.C., Albuquerque Brandão R., Britto B., Cunha de Paula R. \& Ramos Bezerra A.M. 2004. Notes on the Natural History and Conservation Status of Pampas Cat, Oncifelis colocolo, in the Brazilian Cerrado. Mammalia. 68(1): 75-79.

Baker P.J., Harris S., Robertson C.P., Saunders G. \& White P.C. 2004. Is it possible to monitor mammal population changes from counts of road traffic casualties? An analysis using Bristol's red foxes Vulpes vulpes as an example. Mammal Review. 34: 115-130.

Barquez R.M., Días M.M. \& Ojeda R.A. 2006. Mamíferos de Argentina, sistemática y distribución. Sociedad Argentina para el Estudio de los Mamíferos, MendozaArgentina.

Blanco J.C., Corte Y. \& Virgo E. 2005. Wolf response to two kinds of barriers in an agricultural habitat in Spain. Canadian Journal of Zoology. 83: 312-323.

Brodziewska J. 2006. Wildlife tunnels and fauna bridges in Poland: past, present and future, 1997-2013. Páginas 448-460 en: C.L. Irwin, P. Garrett \& K.P. McDermott (editores) Proceedings of the 2005 International Conference on Ecology and Transportation. Center for Transportation and the Environment, North Carolina State University.

Cairo S.L. \& Zalba S.M. 2007. Effects of a paved road on mortality and mobility of red bellied toads (Melanophryniscus sp.) in Argentinean grasslands. Amphibia-Reptilia. 28(3): 377-385.

Caro T.M., Shargei J.A. \& Stoner G.J. 2000. Frequency of medium-sized mammal road-kill in an agricultural landscape in California. American Midland Naturalist. 11: 362-369.

Clarke G.P., White P.C. \& Harris S. 1998. Effects of roads on badger Meles meles populations in south-west England. Biological Conservation. 86: 117-124.

Dellafiore C.M. \& Maceira N.O. 1998. Problemas de conservación de los ciervos autóctonos de la Argentina. Mastozoología Neotropical. 5(2): 137-145.

Dirección Nacional de Vialidad Argentina (DNV). 2010. Informes sobre rutas, tránsito medio anual, y proyecciones del tráfico vehicular. Accesible en: http://www.vialidad.gov.ar

Dodd C.K., Barichivich W.J. \& Smith L.L. 2004. Effectiveness of a barrier wall and culverts in reducing wildlife mortality on a heavily traveled highway in Florida. Biological Conservation. 118: 619-631.

Ferreras P., Gaona P., Palomares F. \& Delibes M. 2001. Restore habitat or reduce mortality? Implications from a population viability analysis of the Iberian lynx. Animal Conservation. 4: 265-274.

Fischer W.A., Ramos-Neto M.B., Silveira L. \& Jacomo A.T. 2004. Human transportation network as ecological barrier for wildlife on Brazilian Pantanal-Cerrado corridors. Páginas 182-194 en: C.L. Irwin, P. Garrett \& K.P. McDermott (editores) Proceedings of the 2003 International Conference on Ecology and Transportation. Center for Transportation and the Environment, NorthCarolina State University.

Forman R.T., Sperling D., Bissonette J.A., Clevenger A.P., Cutshall C.D., Dale V.H., Fahrig L., France R., Goldman C.R., Haenue K., Jones J.A., Swanson F.J., Turrentine T. \& Winter T.C. 2003. Road Ecology: Science and Solutions. Island Press, Washington.

Gittleman J.L., Funk S., MacDonald D. \& Wayne R. 2001. Carnivore Conservation. Conservation Biology. The Zoological Society of London. Cambridge.

Grilo C., Bissonette J.A. \& Santos-Reis M. 2009. Spatialtemporal patterns in Mediterranean carnivore road casualties: Consequences for mitigation. Biological Conservation. 142:301-313.

Iglesias Merchán C. 2007. Corrección de Impactos sobre la Fauna en Infraestructuras del Transporte. Cimbra: Revista del Colegio de Ingenieros Técnicos de Obras Públicas. 377: 30-39.

Luell B.H., Bekker G.J., Cuperus R., Dufek J., Hicks C., Hlavac V., Keller V., Rosell C., Sangwine T., Torslow N. \& Wandall B.M. 2003. Wildlife and Traffic: An European Handbook for Identifying Conflicts and Designing Solutions. KNNV Publishers, UK.

Joyce T.L. \& Mahoney S.P. 2001. Spatial and temporal distributions of moose-vehicle collisions in Newfoundland. Wildlife Society Bulletin. 29: 281-291.

Kerley L.L., Goodrich J.M., Miquelle D.G., Smirnov E.N., Quigley H.B. \& Hornocker M.G. 2002. Effects of roads and human disturbance on Amur Tigers. Conservation Biology. 16(1): 97-108.

Ledec G. \& Posas P.J. 2003. Biodiversity Conservation in Road Projects: Lessons from World Bank Experience in Latin America. Transportation Research Record: Journal of the Transportation Research Board. 1819: 198-202.

Lodé T. 2000. Effect of a motorway on mortality and isolation of wildlife populations. Ambio 29: 163-166.

Malo J.E., Suárez F. \& Díez A. 2004. Can we mitigate animal-vehicle accidents using predictive models? Journal of Applied Ecology. 41: 701-710.

Martino P., Sassaroli J.C., Calvo J., Zapata J. \& Gimeno E. 2008. A mortality survey of free range nutria (Myocastor coypus). European Journal of Wildlife Research. 54: 293-297.

Pereda A. \& Moreno D. 2000. El Venado de las Pampas en Corrientes, diagnóstico de su estado de conservación y propuestas de manejo: Situación crítica. Publicación especial de la Fundación Vida Silvestre Argentina, Buenos Aires. 
Sala E.O., Chapin F.S., Armesto J.J., Berlow E., Bloomfield J., Dirzo R., Huber-Sanwald E., Huenneke L.F, Jackson R.B., Kinzig A., Leemans R., Lodge D.M., Mooney H.A., Oesterheld M., Poff N.L., Sykes M.T., Walker B.H., Walker M. \& Wall D.H. 2000. Global biodiversity scenarios for the year 2100. Science. 287: 1770-1774.

Seiler A. 2005. Predicting locations of moose-vehicle collisions in Sweden. Journal of Applied Ecology. 42: 371-382.figura

Spellerberg I.F. 1998. Ecological effects of roads and traffic: a literature review. Global Ecology \& Biogeography Letters. 7: 317-333.

Strasburg J.L. 2006. Roads and genetic connectivity. Nature 440:875-876.
Taylor B.D. \& Goldingay R.L. 2004. Wildlife road-kills on three major roads in north-eastern New South Wales. Wildlife Research. 31: 83-89.

Trejo A. \& Seijas S. 2003. Una estimación de aves muertas en ruta en el parque nacional Nahuel Huapi, noroeste de la patagonia argentina. Hornero. 18(2): 97-101.

Wilcove D.S., Rothstein D., Dubow J., Phillips A. \& Losos E. 1998. Quantifying threats to imperiled species in the United States. BioScience. 48: 607-615.

Zak M., Cabido M., Cáceres D. \& Díaz S. 2008. What drives accelerated land cover change in central Argentina?. Environmental Management. 42 (2): 181189.

\footnotetext{
${ }^{1}$ Centro de Ecología y Recursos Naturales Renovables, Facultad de Ciencias Exactas Físicas y Naturales, Universidad Nacional de Córdoba, Av. Vélez Sarsfield 1611, CP 5016, Córdoba, Argentina. Teléfono: 03514334141, int. 413. E-mail: fbarri@efn.uncor.edu
} 